\title{
DIRECT INTEGRAL THEORY FOR WEIGHTS, AND THE PLANCHEREL FORMULA ${ }^{1}$
}

\author{
BY COLIN E. SUTHERLAND
}

Communicated by S. Feldman, May, 15, 1973

Our object is to outline the principal results obtained in the study of decomposition theory of weights. We begin by studying decomposition theory for left Hilbert algebras, Tomita algebras, and the associated modular automorphism groups. A decomposition theory for K.M.S. weights is developed together with the necessary existence and uniqueness theorems for these decompositions. We next examine K.M.S. weights on separable $C^{*}$-algebras. As an application we study the Plancherel problem for separable locally compact groups, generalizing the results of [6].

1. Decomposition theory for left Hilbert algebras. Throughout, $\mathfrak{A}$ will denote a left Hilbert algebra with fulfillment $\mathfrak{A}^{\prime \prime}$, Hilbert space completion $\mathscr{H}(\mathfrak{Q})$, left and right regular representations $\pi_{L}$ and $\pi_{R}$, left and right von Neumann algebras $\mathscr{R}_{L}(\mathfrak{U})$ and $\mathscr{R}_{R}(\mathfrak{U})$, sharp operation \#, and corresponding modular operator $\Delta$. (See [4], [5].) Also $\Gamma$ will denote a locally compact space; by a measure $\mu$ on $\Gamma$ we mean a Radon measure.

Definition 1.1. Given $(\Gamma, \mu)$, let $\mathfrak{A}(\gamma)(\gamma \in \Gamma)$ be a left Hilbert algebra. We say $\gamma \mapsto \mathfrak{A}(\gamma)$ is $\mu$-measurable if there are countably many vector fields $\gamma \mapsto \xi_{j}(\gamma), j=1,2, \cdots$ such that:

(i) The fields $\gamma \mapsto \xi_{j}(\gamma)$ are fundamental for (see [4]) the field $\gamma \mapsto$ $\mathscr{H}(\gamma)=\mathscr{H}(\mathfrak{H}(\gamma))$ of Hilbert spaces.

(ii) For $j, k=1,2, \cdots$ the fields $\gamma \mapsto \xi_{j}^{\#}(\gamma)$ and $\gamma \mapsto \xi_{j}(\gamma) \xi_{k}(\gamma)$ are measurable.

(iii) For $\mu$-almost all $\gamma,\left\{\xi_{j}(\gamma): j=1,2, \cdots\right\}$ are dense in $\mathfrak{A}(\gamma)$ with respect to the \#-norm.

Measurability of a field of right Hilbert algebras is defined analogously. Although not necessary for some of our theorems, we restrict to the case where $\Gamma$ is second countable.

AMS (MOS) subject classifications (1970). Primary 46K15; Secondary 46L10, 46L05, 43A10.

${ }^{1}$ The results in this note will form a portion of the authors doctoral dissertation to be submitted at U.C.L.A. in June 1973. The author extends his thanks to his advisor, Professor M. Takesaki, for his extreme patience and helpfulness, and to Professor R. Blattner for discussions on induced representations. 
THEOREM 1.2. If $\gamma \mapsto \mathfrak{A}(\gamma)$ is a measurable field of left Hilbert algebras then $\gamma \mapsto \mathfrak{U}(\gamma)^{\prime}$ is measurable.

Definition 1.3. Let $\{\gamma \mapsto \mathfrak{A}(\gamma)\}$ be a measurable field, and

Define

$$
\mathscr{H}=\int_{\Gamma}^{\oplus} \mathscr{H}(\gamma) d \mu(\gamma)
$$

$$
\mathfrak{U}=\int_{\Gamma}^{\oplus} \mathfrak{A}(\gamma) d u(\gamma)
$$

to be the set of $\xi \in \mathscr{H}$ for which:

(i) If $\xi \sim \xi(\gamma)$ then $\xi(\gamma) \in \mathfrak{A}(\gamma) \mu$-a.e.

(ii) $\gamma \mapsto\left\|\xi^{\#}(\gamma)\right\|^{2}$ is $\mu$-integrable.

(iii) $\gamma \mapsto\left\|\pi_{L}(\xi(\gamma))\right\|$ is essentially bounded.

THeORem 1.4. (i) $\mathfrak{A}$ is a left Hilbert algebra with completion $\mathscr{H}$.

(ii) The field of von Neumann algebras $\gamma \mapsto \mathscr{R}_{L}(\mathfrak{H}(\gamma))$ is measurable, and

$$
\mathscr{R}_{L}(\mathfrak{R})=\int_{\Gamma}^{\oplus} \mathscr{R}_{L}(\mathfrak{H}(\gamma)) d \mu(\gamma) .
$$

(iii) $\mathfrak{A}^{\prime}=\int_{\Gamma}^{\oplus} \mathfrak{A}(\gamma)^{\prime} d \mu(\gamma)$, so that if $\mathfrak{Q}(\gamma)$ is full $\mu$-a.e., then $\mathfrak{A}$ is full.

2. Decomposition theory for Tomita algebras. We are able to give a direct integral theory for Tomita algebras which is fully consistent with the results in $\S 1$ (for example if $\gamma \mapsto \mathfrak{A}(\gamma)$ is a measurable field of full left Hilbert algebras with direct integral $\mathfrak{A}$, and $\gamma \mapsto \mathfrak{A}_{0}(\gamma)$ and $\mathfrak{A}_{0}$ are the corresponding canonical Tomita algebras, then in a very natural sense $\left.\mathfrak{A}_{0}=\int_{\Gamma}^{\oplus} \mathfrak{A}_{0}(\gamma) d \mu(\gamma)\right)$. However, we state only results which will be crucial in the sequel.

Theorem 2.1. Let $\gamma \mapsto \mathfrak{A}(\gamma)$ be a measurable field of full left Hilbert algebras and $\mathfrak{A}=\int_{\Gamma}^{\oplus} \mathfrak{A}(\gamma) d \mu(\gamma)$. Let $\left\{\sigma_{t}: t \in \boldsymbol{R}\right\}$ be the modular automorphism group of $\mathscr{R}_{L}(\mathfrak{R})$ and $\sigma_{t}(\gamma)$ the corresponding group for $\mathscr{R}(\mathfrak{H}(\gamma))$. Then for every $x=\int_{\Gamma}^{\oplus} x(\gamma) d \mu(\gamma)$ in $\mathscr{R}_{L}(\mathfrak{H})$, and every $t \in \boldsymbol{R}, \gamma \mapsto \sigma_{t}(\gamma)(x(\gamma))$ is a measurable operator field and $\sigma_{t}(x)=\int_{\Gamma}^{\oplus} \sigma_{t}(\gamma)(x(\gamma)) d \mu(\gamma)$.

3. Direct integral theory for weights on von Neumann algebras. We make the hypothesis that all von Neumann algebras concerned have separable preduals.

If $\varphi$ is a weight on a von Neumann algebra $\mathscr{M}$, we use the notations $\mathfrak{n}_{\varphi}, \mathfrak{m}_{\varphi}, N_{\varphi}$ as established in [4]; when we say $\varphi$ is quasi-normal we mean that $\varphi\left(\sup _{\alpha \in A} x_{\alpha}\right)=\sup _{\alpha \in A} \varphi\left(x_{\alpha}\right)$ whenever $\left\{x_{\alpha}: \alpha \in A\right\}$ is bounded above in $\mathscr{M}^{+}$; when we say $\varphi$ is normal we mean $\varphi=\sup \left\{\omega: \omega \in \mathscr{M}_{*}, 0 \leqq \omega \leqq \varphi\right\}$.

Definition 3.1. Let $\gamma \mapsto \mathscr{M}(\gamma)(\gamma \in \Gamma)$ be a measurable field of von 
Neumann algebras, and for each $\gamma$ let $\varphi(\gamma)$ be a weight on $\mathscr{M}(\gamma)$. We say $\gamma \mapsto \varphi(\gamma)$ is measurable if

(i) there exist measurable operator fields $\gamma \rightarrow x_{j}(\gamma), j=1,2, \cdots$, with $x_{j}(\gamma) \in \mathfrak{n}(\gamma)=\mathfrak{n}_{\varphi(\gamma)}$ and $\left\{x_{j}(\gamma): j=1,2, \cdots\right\} \sigma$-weakly dense in $\mathfrak{n}(\gamma)$;

(ii) there exist measurable operator fields $\gamma \mapsto y_{j}(\gamma), j=1,2, \cdots$, with $x_{j}(\gamma) \in \mathfrak{n}(\gamma) \cap \mathfrak{n}(\gamma)^{*}$ such that $\left\{y_{j}(\gamma): j=1,2, \cdots\right\}$ is dense in $\mathfrak{n}(\gamma) \cap \mathfrak{n}(\gamma)^{*}$ with respect to the metric

$$
d(\gamma)\left(z_{1}, z_{2}\right)=\varphi(\gamma)\left(\left(z_{1}-z_{2}\right)\left(z_{1}-z_{2}\right)^{*}+\left(z_{1}-z_{2}\right)^{*}\left(z_{1}-z_{2}\right)\right)^{1 / 2} .
$$

(iii) If $\gamma \mapsto x(\gamma) \in \mathscr{M}(\gamma)^{+}$is measurable, then $\gamma \mapsto \varphi(\gamma)(x(\gamma))$ is measurable.

If $\gamma \mapsto \varphi(\gamma)$ satisfies 3.1(i) and 3.1(iii) we say $\gamma \mapsto \varphi(\gamma)$ is weakly measurable.

DEFINITION 3.2. Let $\gamma \mapsto \varphi(\gamma)$ be weakly measurable as above, and $\mathscr{M}=\int_{\Gamma}^{\oplus} \mathscr{M}(\gamma) d \mu(\gamma)$. Define $\varphi=\int_{\Gamma}^{\oplus} \varphi(\gamma) d \mu(\gamma)$ on $\mathscr{M}$ by

$$
\varphi\left(\int_{\Gamma}^{\oplus} x(\gamma) d \mu(\gamma)\right)=\int_{\Gamma}^{\oplus} \varphi(\gamma)(x(\gamma)) d \mu(\gamma) .
$$

It is clear that $\varphi$ is a weight, and readily verified that the properties of quasi-normality, $\sigma$-weak lower semicontinuity, normality and semifiniteness are preserved under the direct integral.

Hereafter, (almost) all weights will be faithful, normal and semifinite; we omit the qualification. Suppose $\gamma \mapsto \varphi(\gamma)$ is an arbitrary field of weights; let $\mathfrak{A}(\gamma)$ be the full left Hilbert algebra associated with $\varphi(\gamma)$, and $\Lambda(\gamma)=$ $\left\{\omega \in \mathscr{M}(\gamma)_{*}^{+}: \omega \leqq \varphi(\gamma)\right\}$.

Theorem 3.3. Suppose $\gamma \mapsto \mathscr{M}(\gamma)$ is a measurable field of Von Neumann algebras, and $\varphi(\gamma)$ is a weight on $\mathscr{M}(\gamma)$. Then the following are equivalent.

(i) $\gamma \mapsto \varphi(\gamma)$ is measurable.

(ii) $\gamma \mapsto \mathfrak{A}(\gamma)$ is measurable.

(iii) There exist measurable cross-sections $\gamma \mapsto \omega_{j}(\gamma)$ of $\gamma \mapsto \Lambda(\gamma), j=$ $1,2, \cdots$, such that

(a) there exists a $\mu$-null set $N \subset \Gamma$ such that, for $\gamma \in \Gamma-N$ and $z \in \mathscr{M}(\gamma)^{+}$, $\varphi(\gamma)(z)=\sup \left\{\omega_{j}(\gamma)(z): j=1,2, \cdots\right\}$, and

(b) for each $j_{1}, j_{2}=1,2, \cdots$ and $\varepsilon>0$, there exists $k$ such that $\omega_{j_{i}}(\gamma) \leqq$ $(1+\varepsilon) \omega_{k}(\gamma) \mu$-a.e., $i=1,2$.

In the course of the proof of 3.3 we show

THEOREM 3.4. Let $\gamma \mapsto \mathfrak{A}(\gamma)$ be a measurable field of full left Hilbert algebras, with direct integral $\mathfrak{A}$, and $\varphi(\gamma), \varphi$ be the canonical weights on $\mathfrak{U}(\gamma)$, 升. Then $\varphi=\int_{\Gamma}^{\oplus} \varphi(\gamma) d \mu(\gamma)$. 
4. Existence and uniqueness theorems. The basic result here is as follows (see [1], [2]).

THEOREM 4.1. Suppose $\mathscr{M}=\mathscr{R}_{L}(\mathfrak{U})$. Then $\mathscr{M}$ is decomposable over $(\Gamma, \mu)$ if and only if there is a measurable field $\gamma \mapsto \mathfrak{U}(\gamma)(\gamma \in \Gamma)$ with $\mathfrak{U}=\int_{\Gamma}^{\oplus} \mathfrak{A}(\gamma) d \mu(\gamma)$.

The decomposition theorems for K.M.S. weights and Tomita algebras follow automatically from Theorems 4.1, 3.4 and \$2. For measurable fields of full left Hilbert algebras we obtain an "essential uniqueness" theorem (up to measure isomorphism), and for weights we have the following

THEOREM 4.2. If $\gamma \mapsto \varphi_{1}(\gamma), \gamma \mapsto \varphi_{2}(\gamma)$ are weakly measurable and measurable fields of weights respectively and $\int_{\Gamma}^{\oplus} \varphi_{1}(\gamma) d \mu(\gamma)=\int_{\Gamma}^{\oplus} \varphi_{2}(\gamma) d \mu(\gamma)$, then $\varphi_{1}(\gamma)=\varphi_{2}(\gamma) \mu$-a.e.

5. Decompositions of K.M.S. weights on $C^{*}$-algebras. Let $A$ be a separable $C^{*}$-algebra, and $\varphi$ a faithful semifinite weight on $A$, K.M.S. for the one-parameter automorphism group $\sigma_{t}$ on $A$ [4]. Let $\pi_{\varphi}$ be the corresponding representation of $A$.

THEOREM 5.1. Suppose $\pi_{\varphi}=\int_{\Gamma}^{\oplus} \pi(\gamma) d \mu(\gamma)$. Then there is a family $\{\varphi(\gamma): \gamma \in \Gamma\}$ of weights on $A$ such that

(i) for $x \in A^{+}, \gamma \mapsto \varphi(\gamma)(x)$ is measurable and $\varphi(x)=\int_{\Gamma} \varphi(\gamma)(x) d \mu(\gamma)$, and

(ii) for each $\gamma \in \Gamma, \varphi(\gamma)$ satisfies the K.M.S. condition for $\sigma_{t}$.

We also show that any lower semicontinuous weight on $A$ may be approximated from below by a countable family of positive linear functionals.

6. Applications to the Plancherel theorem. Let $G$ be a separable locally compact topological group, with left regular representation $\lambda^{G}$ on $L^{2}(G)=\mathscr{G}$. Considering $\mathscr{M}(G)=\left\{\lambda^{G}(g): g \in G\right\}^{\prime \prime}$ and the canonical weight $\varphi$ on $\mathscr{M}(G)$, we may, by the remarks following 4.1 , write

$$
\{\mathscr{M}(G), \varphi\}=\int_{\Gamma \theta}^{\oplus}\{\mathscr{M}(\gamma), \varphi(\gamma)\} d \mu(\gamma),
$$

where $\Gamma_{G}$ is the spectrum of the center $\mathscr{Z}(G)$ of $\mathscr{M}(G)$, and the decomposition is central. We may regard this as a Plancherel formula for $G$, and our concern is to obtain more precise information concerning $\Gamma_{G}$, and the components $\varphi(\gamma)$. To this end, put $H=\left\{g \in G: \delta_{G}(g)=1\right\}$, where $\delta_{G}$ is the modular function of $G$. Let $t \mapsto \sigma_{t}^{\varphi}$ be the modular automorphism group for $\varphi$. 
Theorem 6.1. Put $\mathscr{M}_{0}(G)=\left\{x \in \mathscr{M}(G): \sigma_{t}^{\varphi}(x)=x, t \in \mathbf{R}\right\}$. Then

$$
\mathscr{M}_{0}(G)=\left\{\lambda^{G}(h): h \in H\right\}^{\prime \prime} .
$$

We use the notation $\mathscr{M}(H), \lambda^{H}, \mathscr{H}=L^{2}(H), \mathscr{Z}(H)$ in the obvious way, and let $\tau$ be the canonical trace on $\mathscr{M}(H)$. We denote by $C^{*}(G)$ the group $C^{*}$-algebra of $G$, and by $\pi^{G}$ the usual representation of $C^{*}(G)$ in $\mathscr{M}(G)$. Similarly for $H$. Also $\mathscr{A} \subset \mathscr{L}(\mathscr{G})$ will denote the canonical system of imprimitivity associated with the induction of $\lambda^{H}$ to $\lambda^{G}$ [3]. Note that by 6.1 we may regard $\mathscr{Z}(G)$ as a subalgebra of $\mathscr{Z}(H)\left(\right.$ since $\mathscr{Z}(G) \subset \mathscr{M}_{0}(G)$ ).

We consider the following two central decompositions

$$
\begin{aligned}
\left\{\mathscr{M}(G), \lambda^{G}, \varphi, \mathscr{A}_{H}, \mathscr{G}, \pi^{G}\right\} \\
\quad=\int_{\Gamma_{G}}^{\oplus}\left\{\mathscr{M}(\omega), \lambda^{G}(\omega), \varphi(\omega), \mathscr{A}_{H}(\omega), \mathscr{G}(\omega), \pi^{G}(\omega)\right\} d \mu(\omega) .
\end{aligned}
$$

(ii) $\left\{\mathscr{M}(H), \lambda^{H}, \tau, \mathscr{H}, \pi^{H}\right\}=\int_{\Gamma H}^{\oplus}\left\{M(\gamma), \lambda^{H}(\gamma), \tau(\gamma), \mathscr{H}(\gamma), \pi^{H}(\gamma)\right\} d \nu(\gamma)$.

Note that $G$ acts on $H$ via inner automorphisms, and the induced action of $G$ on $\Gamma_{H}$ is Borel. Thus we may perform the ergodic decomposition $\nu=\int_{X} v(\zeta) d m(\zeta)$ and so, from (ii), write

$$
\left\{\mathscr{M}(H), \lambda^{H}, \tau, \mathscr{H}, \pi^{H}\right\}=\int_{X}^{\oplus}\left\{M(\zeta), \lambda^{H}(\zeta), \tau(\zeta), \mathscr{H}(\zeta), \pi^{H}(\zeta)\right\} d m(\zeta) .
$$

TheOREM 6.2. (i) $\mathscr{A}_{H}(\omega)$ is a system of imprimitivity for $G$ over $H$, $\mu$-a.e.

(ii) there is a measure isomorphism $\beta:(X, m) \mapsto\left(\Gamma_{G}, \mu\right)$ such that

$$
\left\{\lambda^{G}(\beta(\zeta)), \mathscr{G}(\beta(\zeta))\right\} \text { and } \operatorname{Ind}_{H}^{G}\left\{\lambda^{H}(\zeta), \mathscr{H}(\zeta)\right\}
$$

are unitarily equivalent.

Thus we identify $(X, m)$ and $\left(\Gamma_{G}, \mu\right)$. Note that this theorem extends the work of Tatsuuma [6], who deals with the case when the orbit space $\Gamma_{H} / G$ is countably separated. Finally we show how to relate the weights $\varphi(\omega)$ and $\tau(\omega)\left(\omega \in \Gamma_{G}\right)$.

Let $\mathscr{K}(G)(\mathscr{K}(H))$ denote the spaces of continuous complex valued functions on $G(H)$ with compact support, endowed with the usual Tomita algebra structure. For $\xi \in \mathscr{K}(G)$ we may write $(\S 3) \xi \sim \xi(\omega)$ and $\pi_{L}(\xi) \sim$ $\pi_{L}(\xi(\omega))$. Define bilinear form $\chi_{G}(\omega)$ on $\mathscr{K}(G) \times \mathscr{K}(G)$ by

$$
\chi_{G}(\omega)(\xi, \eta)=\varphi(\omega)\left(\pi_{L}\left(\eta^{\#} * \xi\right)(\omega)\right) .
$$

A bilinear functional $\chi_{H}(\omega)$ is defined on $\mathscr{K}(H) \times \mathscr{K}(H)$ analogously. 
Since $\xi(\omega) \in \mathscr{G}(\omega) \mu$-a.e., and $\mathscr{G}(\omega)$ is the Hilbert space of the representation of $G$ induced from $\lambda^{H}(\omega)$, we may identify $\xi(\omega)$ with a map $\xi(\omega): g \in$ $G \mapsto \xi(\omega)(g) \in \mathscr{H}(\omega)$.

THEOREM 6.3. Statements are to be interpreted "almost" everywhere.

(i) For $\xi \in \mathscr{K}(G)$ and $g \in G, \tilde{\xi}(\omega)(g)$ is in the domain of $\chi_{H}(\omega)$, and $\omega \mapsto \chi_{H}(\omega)(\xi(\omega)(g), \tilde{\xi}(\omega)(g))$ is measurable.

(ii) For $\xi \in \mathscr{K}(G)$ and $\omega \in \Gamma_{G}$, the map $g \mapsto \chi_{H}(\omega)(\xi(\omega)(g), \tilde{\xi}(\omega)(g))$ is constant on the cosets of $H$, and measurable on $G / H$.

(iii) The identification $\xi(\omega) \mapsto \tilde{\xi}(\omega)$ may be made so that

$$
\chi_{G}(\omega)(\xi, \xi)=\int_{G / H} \chi_{H}(\omega)(\xi(\omega)(g), \tilde{\xi}(\omega)(g)) d \alpha(\dot{g}),
$$

where $\alpha$ is a Haar measure on $G / H \subset R_{+}^{*}$.

(iv) For $\xi \in \mathscr{K}(G),\|\xi\|_{G}^{2}=\int_{\Gamma_{G}} \chi_{G}(\omega)(\xi, \xi) d \mu(\omega)$.

(v) The functionals $\chi_{H}(\omega), \chi_{G}(\omega)$ may be extended to dense left ideals in $C^{*}(H), C^{*}(G)$ in such a way that the associated representations of $C^{*}(H)$ and $C^{*}(G)$, respectively, are unitarily equivalent to $\pi^{H}(\omega)$ and $\pi^{G}(\omega)$.

\section{REFERENCES}

1. J. Dixmier, Algebres quasi-unitaire, Comment Math. Helv. 26 (1952), 275-322. MR 14, 660.

2. —_, Algebres des operateurs dans l'espace Hilbertien, 2nd ed., Gauthier-Villars, Paris, 1969.

3. G. W. Mackey, Unitary representations of group extensions. I, Acta Math. 99 (1958), 265-311. MR 20 \#4789.

4. M. Takesaki, Theory of operator algebras, U.C.L.A. Lecture Notes, 1969.

5. G. K. Pederson and M. Takesaki, The Radon Nikodym theorem for von Neumann algebras, Acta Math. 130 (1973), 53-87.

6. N. Tatsuuma, Plancherel formula for non-unimodular locally compact groups, J. Math. Kyoto Univ. 12 (1972).

Department of Mathematics, University of California, los Angeles, CaliFORNIA 90024 